\title{
Protease inhibitor phenotype BsaskatoonM is not associated with emphysema - A 20-year follow-up study
}

\author{
Ruzica Jokic MD ${ }^{1}$, Kendra L Fisher MD FRCPC ${ }^{2}$, Donald W Cockcroft MD FRCPC ${ }^{1}$ \\ ${ }^{1}$ Division of Respiratory Medicine and ${ }^{2}$ Department of Medical Imaging, \\ University of Saskatchewan, Saskatoon, Saskatchewan
}

\begin{abstract}
R Jokic, KL Fisher, DW Cockcroft. Protease inhibitor phenotype BsaskatoonM is not associated with emphysema - A 20-year follow-up study. Can Respir J 1999;6(5):407-411.
\end{abstract}

OBJECTIVE: To investigate the association between the PiBsaskatoon variant of alpha-1 protease inhibitor $(\alpha 1 \mathrm{Pi})$, present in the heterozygous state and the development of emphysema.

DESIGN: Twenty-year follow-up in the third generation of a family with the variant, naturally controlled with regard to both environmental influences and other genetic factors.

SETTING: University teaching hospital.

POPULATION STUDIED: Ten siblings, five with PiBsaskatoonM phenotype and five with PiM phenotype, 33 to 46 years of age.

INTERVENTIONS: Respiratory symptoms and smoking histories; pulmonary function tests, including static lung volumes, dynamic lung volumes before and after salbutamol $200 \mu \mathrm{g}$, and diffusing capacity; allergen prick skin tests; serum $\alpha 1$ Pi level, chest radiographs and high resolution computerized tomography lung scans

MAIN RESULTS: The two groups of siblings had similar mean ages, smoking histories and prevalence of current mild respiratory symptoms. Pulmonary function data showed normal mean values and no statistically significant differences for all the variables between the two groups. Chest radiographs were normal in all subjects. High resolution computerized tomography scans were normal in eight subjects, and demonstrated mild and very mild centrilobular emphysema in the two subjects with greatest smoking histories (approximately 30 pack-year each); both of these were PiBsaskatoonM phenotype.

CONCLUSION: There is no evidence of an association between $\alpha 1$ Pi phenotype PiBsaskatoonM and the development of emphysema.

Key Words: Alpha-1 protease inhibitors; Emphysema; Pi Bsaskatoon

\section{Le phénotype BsaskatoonM de l'inhibiteur de} la protéase n'est pas associé à l'emphysème une étude de suivi de 20 années

OBJECTIF : Rechercher une association entre le variant PiBsaskatoon de l'inhibiteur de la protéase alpha-1 ( $\alpha 1 \mathrm{Pi})$ présent à l'état hétérozygote, et le développement de l'emphysème.

MODÈLE : Un suivi de vingt ans dans la troisième génération d'une famille porteuse du variant et naturellement contrôlée à 
la fois pour ce qui est des influences environnementales et des autres facteurs génétiques.

CONTEXTE : Hôpital universitaire.

POPULATION ÉTUDIÉE : Dix membres d'une même fratrie dont cinq porteurs du phénotype PiBsaskatoonM et cinq autres du phénotype PiM, âgés entre 33 et 46 ans.

INTERVENTIONS : Symptômes respiratoires et antécédents de tabagisme ; épreuves de fonction respiratoire, y compris volumes pulmonaires statiques, volumes pulmonaires dynamiques avant et après l'administration de $200 \mu \mathrm{g}$ de salbutamol, ainsi que la capacité de diffusion pulmonaire ; tests cutanés (prick-test) avec des allergènes, niveau du $\alpha 1$ Pi dans le sérum, radiographies pulmonaires et tomographie pulmonaire à haute résolution.

PRINCIPAUX RÉSULTATS : L'âge moyen, les antécé- dents de tabagisme et la prévalence de symptômes respiratoires légers et présents au moment de l'étude étaient similaires dans les deux groupes étudiés. Les données sur la fonction respiratoire indiquaient des valeurs moyennes normales, sans différence statistique significative pour l'ensemble des variables entre les deux groupes. Les radiographies pulmonaires étaient normales chez tous les sujets. Les tomographies pulmonaires à haute résolution étaient normales chez huit sujets, et révélaient un emphysème centrolobulaire, léger à très léger, chez les deux sujets avec des antécédents de tabagisme plus prononcé (environ 30 paquets/année pour chacun) ; ces deux sujets étaient porteurs du phénotype PiBsaskatoonM.

CONCLUSION : Il n'y a aucune preuve d'une association entre le phénotype PiBsaskatoonM de l'inhibiteur de la protéase alpha-1 ( $\alpha 1 \mathrm{Pi})$ et le développement de l'emphysème.
$\mathrm{S}_{\mathrm{e}}^{\mathrm{e}}$ erum alpha-1 proteinase inhibitor ( $\alpha 1 \mathrm{Pi}$, formerly known as alpha-1 antitrypsin) deficiency is a condition that predisposes individuals to the development of emphysema (1). In 1979, a new anodal $\alpha 1$ Pi variant was identified in our institution in the proposita, a 48-year-old woman with early onset severe panlobular pulmonary emphysema suspected of Pi deficiency (2) (Figure 1, II-9). The new variant designated PiBsaskatoon was present in the heterozygous state (BsaskatoonM) in three generations of the family, and was associated with normal serum $\alpha 1$ Pi levels and normal in vitro elastase inhibitory capacity (2). Although these find- ings suggested that PiBsaskatoon should not predispose individuals to emphysema, the other three BsaskatoonM members from the first two generations of this family had documented $(n=2)$ or reported $(n=1)$ emphysema, as opposed to none of the nonaffected family members (Figure 1).

The availability of 11 children (five PiBsaskatoonM and six PiM phenotype), now approaching the age when their mother had advanced emphysema, allowed us to perform an investigation that was naturally controlled with regard to both environmental influences and other genetic factors.

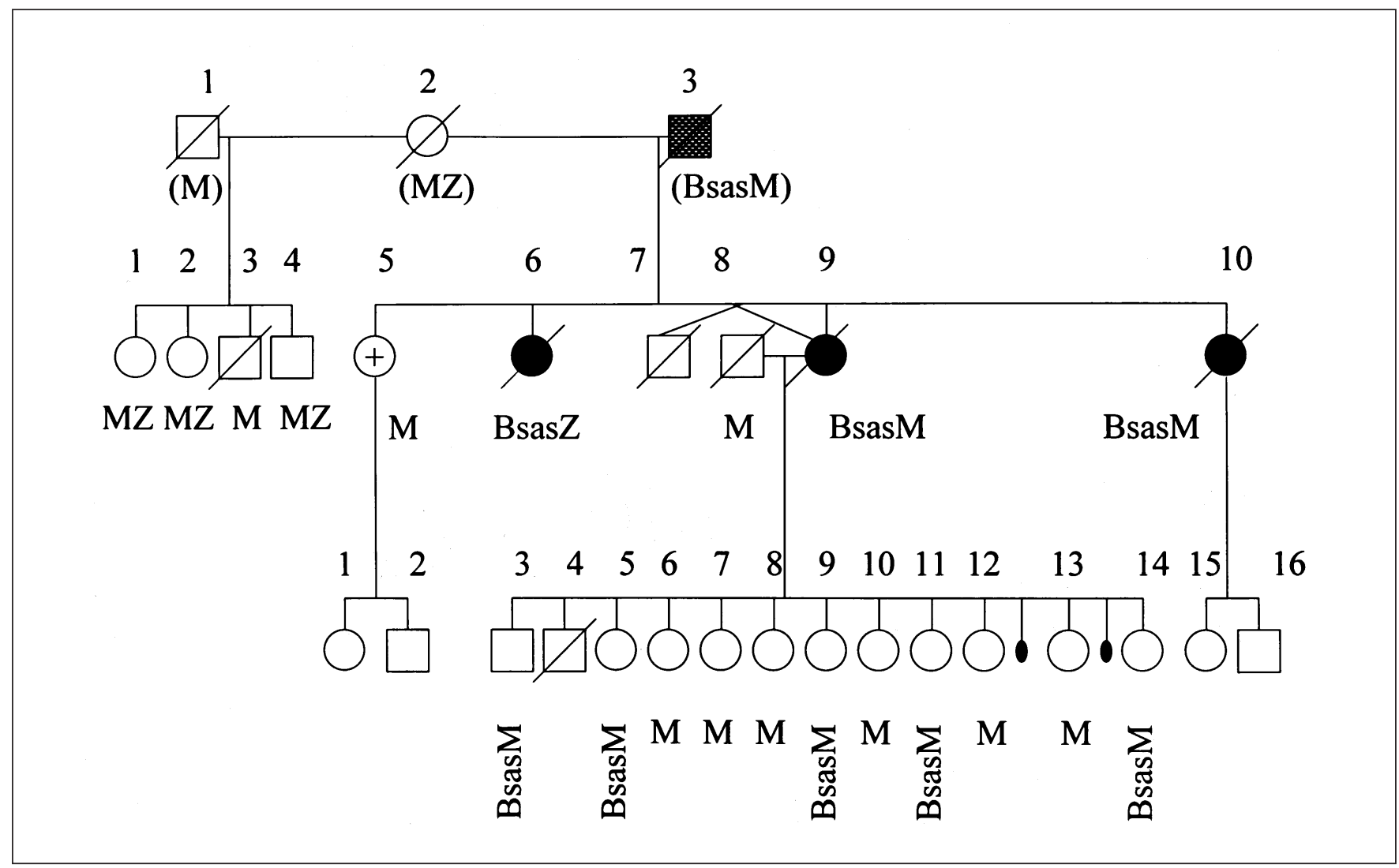

Figure 1) Pedigree showing al Pi phenotypes and family members with emphysema $(\square$ emphysema; normal; $\square$ deceased; still birth/abortion; ( ) presumed phenotype). Modified and reprinted with permission from reference 2 


\section{SUBJECTS AND METHODS}

Subjects: Ten of the 11 siblings, five with PiBsaskatoonM phenotype and five with the PiM phenotype, now 33 to 46 years of age, participated in this follow-up study (III-7, a 42year-old female with PiM phenotype moved out of the province and was not available for testing).

Investigations: Respiratory symptoms and smoking histories were recorded using the standardized American Thoracic Society (ATS) questionnaire (3).

Pulmonary function tests, including static lung volumes, dynamic lung volumes before and after salbutamol $200 \mu \mathrm{g}$, and diffusing capacity were measured on each subject (6200 Automated Body Plethysmograph, Sensormedics, Yorba Linda, California). The severity of lung function abnormalities and the bronchodilator response were assessed according to the ATS criteria (4). A standardized battery of 25 allergen prick skin tests were performed on each subject.

Serum $\alpha 1$ Pi was quantified using the immunonephalometric assay with antibodies directed to human $\alpha 1 \mathrm{Pi}$ (Beckman Array 360 Analyzer, Beckman Instruments, Brea, California). The samples were obtained when the subjects were not pregnant, or suffering from respiratory infection or other significant illnesses. Two subjects were on long term contraceptive treatment with depo-medroxyprogesterone (Depo-Provera, Pharmacia \& Upjohn, Mississauga, Ontario).

A chest radiograph (posterior anterior and lateral projections) and high resolution computerized tomography (CT) lung scans (eight $1 \mathrm{~mm}$ thick slices every $30 \mathrm{~mm}$ through the lungs during full inspiration) were obtained in all subjects. The clinical radiologist was blinded to the subjects' phenotype while analyzing the radiographs and CTs.

\section{RESULTS}

The demographic and clinical characteristics of the two groups of siblings are presented in Table 1. The PiBsaskatoonM and the PiM groups had similar mean ages and smoking histories (all five subjects in the PiBsaskatoonM group were exsmokers, whereas two of four subjects with a smoking history in the PiM group were current smokers). All 10 subjects were atopic (at least one skin test was positive, with a wheal size $2 \mathrm{~mm}$ or more larger than the negative control). The presence of current mild respiratory symptoms within
TABLE 1

Demographic and clinical characteristics

\begin{tabular}{lcc}
\hline & $\begin{array}{c}\text { PiBsaskatoon } \mathbf{M} \\
(\mathbf{n}=5)\end{array}$ & PiM \\
\hline Age (years) (mean \pm SD) & $39.2 \pm 6.1$ & $38 \pm 4.1$ \\
Sex (male:female) & $1: 4$ & $0: 5$ \\
Smoking (n) & 5 & 4 \\
Pack-years (mean \pm SD) & $18.3 \pm 12.8$ & $14.7 \pm 8.5$ \\
Atopy ( $n$ ) & 5 & 5 \\
Positive skin tests ( $n$ ) & $2.8 \pm 0.8$ & $2.8 \pm 2.4$ \\
$\quad$ (mean \pm SD) & & \\
Respiratory symptoms (n) & 3 & 2 \\
Asthma & 3 & 2 \\
Hay fever & 2 & 1 \\
Alpha-1 protease inhibitor & $1.42 \pm 0.25$ & $1.43 \pm 0.1$ \\
$\quad$ (g/L) (normal range 0.8-2.0) & & \\
(mean \pm SD) & & \\
\hline
\end{tabular}

the two groups (wheezing, cough, episodes of increased cough and phlegm) was also similar among the groups. Three subjects in the PiBsaskatoonM group and two of five subjects in the PiM group reported being diagnosed with asthma. Only one subject (III-9) used inhaled beta2-agonists occasionally; no one used any other respiratory treatment.

Pulmonary function data showed normal mean values and no statistically significant differences for all the variables between the two groups (Table 2). All 10 subjects had normal diffusing capacities (70\% predicted or more). In the PiBsaskatoonM group there were two subjectswith mild obstructive airway dysfunction and a positive $(12 \%$ or more increase in forced expiratory volume in $1 \mathrm{~s}\left[\mathrm{FEV}_{1}\right]$ ) bronchodilator response, and in the PiM group, there was one subject with this dysfunction. Mild to moderate lung hyperinflation, as assessed by total lung capacity or residual volume greater than $120 \%$ predicted (5) was present in four of five individuals in the PiBsaskatoonM group and one of five in the PiM group.

Chest radiographs were normal in all subjects. High resolution CT scans demonstrated mild (III-5) and very mild (III-11) centrilobular emphysema in the two subjects with PiBsaskatoonM phenotype, respectively. These participants had the greatest smoking histories (approximately 30 packyear each).

TABLE 2

Lung function tests

\begin{tabular}{lccc}
\hline & PiBsaskatoonM (n=5) & PiM $(\mathbf{n}=5)$ & P \\
\hline Forced vital capacity (FVC) (\% predicted) & $113.4 \pm 6.0$ & $110.8 \pm 12.0$ & 0.68 \\
Forced expiratory volume in 1s (FEV $)$ (\% predicted) & $104.2 \pm 10.5$ & $101.7 \pm 20.0$ & 0.84 \\
$\% \Delta \mathrm{FEV}_{1}$ & $11.2 \pm 6.6$ & $7.2 \pm 9.2$ & 0.45 \\
FEV 1 FVC $(\%)$ & $71.2 \pm 7.6$ & $68.6 \pm 7.1$ & 0.59 \\
Total lung capacity (\% predicted) & $124.6 \pm 11.6$ & $116.0 \pm 9.5$ & 0.24 \\
Residual volume (\% predicted) & $135.6 \pm 25.9$ & $111.0 \pm 17.5$ & 0.12 \\
Functional residual capacity (\% predicted) & $117.2 \pm 15.7$ & $109.2 \pm 22.0$ & 0.53 \\
Carbon monoxide diffusing capacity of the lungs (\% predicted) & $94.4 \pm 6.7$ & $83.8 \pm 9.4$ & 0.07 \\
\hline
\end{tabular}

Data presented as mean $\pm S D$ 


\section{DISCUSSION}

This follow-up study in a family with PiBsaskatoon variant of $\alpha 1$ Pi failed to demonstrate an association between this phenotype and the development of emphysema. The study was performed in two groups of siblings with similar demographic and clinical characteristics, 20 years after the initial assessments, at the age when their mother had symptoms and the clinical manifestations in cases of $\alpha 1$ Pi deficiency or impaired function would be expected to appear.

The results of this study show no significant differences in the symptoms, lung function tests and radiographic findings between subjects with PiBsaskatoonM phenotype and the PiM phenotype. Mild respiratory symptoms and mild airflow obstruction were documented among the family members with both phenotypes. These findings were not a surprise because the subjects often had a history of smoking, allergies and/or asthma. Significant breathing difficulties and lung function tests that suggest emphysema (reduced $\mathrm{FEV}_{1}$ :forced vital capacity (FVC) ratio, evidence of air trapping and a low gas transfer factor) were not documented in this family. Consistent with the findings of the original investigation (2), PiBsaskatoonM phenotype was not associated with low serum $\alpha 1$ Pi levels.

Two of five subjects with PiBsaskatoon variant had radiographic evidence of very mild centrilobular emphysema, a finding common in 30 pack-year smokers (the two subjects had the greatest smoking histories: 28.8 and 29.4 pack-years, respectively). However, the pulmonary function tests including diffusing capacities and chest radiographs were normal; thus, the CT scans showing very mild centrilobular emphysema are not likely to be of any clinical significance. Chest radiographs and high resolution CT lung scans of $\alpha 1 \mathrm{Pi}$ deficiency-related emphysema typically show bilateral predominant basal emphysema with paucity and pruning of the pulmonary vessels compared with the centrilobular upper lobe disease seen in smokers (1).

According to the protease-antiprotease hypothesis, emphysema results from destruction of the connective tissue matrix of alveolar walls by proteolytic enzymes released by inflammatory cells in the alveoli. $\alpha 1 \mathrm{Pi}$ is the principal serum inhibitor of proteolytic enzymes such as neutrophil elastase $(5,6)$. Over 90 different genetically determined variants of $\alpha 1 \mathrm{Pi}$ have been described, known as the protease inhibitor system. $\alpha 1 \mathrm{Pi}$ is transmitted in a codominant fashion, ie, the gene product from each parent is expressed in the offspring, and deficiency occurs as a result of inheritance of two protease inhibitor deficiency alleles on the chromosomal segment 14q32.1 (7). The most common deficiency allele that results in severe $\alpha 1$ Pi deficiency is PiZ $(1,7,8)$. Some other rare deficiency alleles have also been demonstrated to produce a serum concentration $\alpha 1$ Pi below a so-called protective threshold value, increasing the risk of emphysema $(8,9)$. Mutations that result in the complete or almost complete absence of $\alpha 1-\mathrm{Pi}$ in the serum are rare (10). However, as shown by studies in this and other families $(11,12)$, most variants of the $\alpha 1 \mathrm{Pi}$ are not associated with changes in the $\alpha 1 \mathrm{Pi}$ serum concentration or changes in the inhibitory activity against proteolytic enzymes.

There are several possible explanations for the presence of emphysema in the previous two generations of this family with the PiBsaskatoonM phenotype. The most plausible explanation is chance occurrence due to the presence of a common environmental factor (ie, smoking). All family members with the PiBsaskatoonM phenotype and documented emphysema were smokers. Indeed, it is known that the majority of patients develop emphysema as a consequence of smoking, and only $1 \%$ to $2 \%$ develop the condition as a result of genetic deficiency of the plasma protease inhibitors (7). In subjects with the $\alpha 1$ Pi deficiency, it has been documented that cigarette smoking accelerates the onset of dyspnea, among severely deficient individuals by as much as 19 years (8).

Although recent epidemiological studies show a high prevalence of $\alpha 1$ Pi deficiency (in the case of PiZ phenotype close to one of 3000 Americans), only a minority of individuals affected with $\alpha 1$ Pi deficiency are currently recognized by the medical community (8). The association between a possible genetic cause and emphysema may carry significant prognostic consequences and is important for other family members. The genetic cause of the $\alpha 1$ Pi deficiency is usually suspected in a patient with emphysema under the age of 50 years, with little or no smoking history, with a suggestive family history or both. Thus, when choosing a family in which the search for a possible new variant is being conducted, selection bias is likely to occur. Although the proposita (II-9) had a 25 pack-year smoking history, the early onset of symptoms, the relatively low smoking history and the presence of emphysema in two generations prompted further investigation, and thus the discovery of the new $\alpha 1$ Pi variant in this family.

Cook and colleagues (13) have recently studied a 31year-old woman with severe irreversible airflow limitation suspected of protease inhibitor deficiency, who was subsequently diagnosed with a tracheal polyp and whose lung function tests returned to normal after polyp removal. A rare $\alpha 1$ Pi phenotype Pi EFranklinS was determined. Similar to PiBsaskatoon variant, the serum $\alpha 1 \mathrm{Pi}$ and anti-neutrophil elastase capacity of this variant were normal. However, the association rate constant of the isolated and purified PiEFranklin protein was significantly lower than that in the normal $\mathrm{M}$ variant. This implied that this form of $\alpha 1 \mathrm{Pi}$ is expressed at normal levels in serum but is functionally impaired as an inhibitor of human neutrophil elastase. However, sufficient molecules of $\alpha 1 \mathrm{Pi}$ in the serum achieved $100 \%$ inhibition of elastase (13). Although we have not investigated the association rate constant of the isolated and purified PiBsaskatoon, it can be postulated that normal serum concentrations of this protease inhibitor provide adequate protection against proteolytic enzymes, due in part to the presence of $\mathrm{M}$ protein in the heterozygotes. Thus, subjects heterozygous for this rare variant appear to have little risk for the development of emphysema. We have not had the opportunity to study subjects homozygous for Pi Bsaskatoon.

Future investigations in other families with PiBsaskatoon 
$\alpha 1$ Pi phenotype will be able to show whether it should be regarded as a possible dysfunctional allele and whether people with any heterozygous combination with either $\mathrm{Z}$ or Null allele should be regarded as a theoretical risk of developing emphysema.

ACKNOWLEDGEMENTS: Dr Jokic is a research fellow supported by Boehringer Ingelheim Canada.

\section{REFERENCES}

1. Mahadeva R, Lomas DA.Genetics and respiratory disease. 2. Alpha 1-antitrypsin deficiency, cirrhosis and emphysema. Thorax 1998;53:501-5.

2. Horne SL, Tennent RK, Cockcroft DW. A new anodal alpha-1 antitrypsin variant associated with emphysema: Pi Bsaskatoon. Am Rev Respir Dis 1982;125:594-600.

3. Ferris BG. Recommended respiratory questionnaire for use with adults and children in epidemiological research. Am Rev Respir Dis 1978;118(Suppl 1):7-47.

4. American Thoracic Society. Workshop on Lung Function testing: Selection of Reference Values and Interpretative Strategies. Am Rev Respir Dis 1991;144:1202-18.
5. Carrell RW, Jeppsson JO, Laurell CB, et al. Structure and variation of human $\alpha 1$ antitrypsin. Nature 1982;298:329-34.

6. Norman MR, Mowat AP, Hutchison DC. Molecular basis, clinical consequences and diagnosis of alpha-1 antitrypsin deficiency. Ann Clin Biochem 1997;34:230-46.

7. Alpha 1-antitrypsin deficiency: Memorandum from a WHO meeting. Bull World Health Organ 1997;75:397-415 .

8. Stoller JK. Clinical features and natural history of severe alpha 1-antitrypsin deficiency. Roger S. Mitchell Lecture. Chest 1997;111(Suppl 6):123S-8S.

9. Cox DW, Billingsley G. Rare deficiency types of $\alpha 1$ antitrypsin. Electrophoretic variation and DNA haplotypes. Am J Hum Genet 1989;44:844-54.

10. Cook L, Janus ED, Brenton S, Tai E, Burdon J. Absence of alpha-1-antitrypsin (Pi Null Bellingham) and the early onset of emphysema. Aust NZ J Med 1994;24:263-9.

11. Cook L, Burdon J, Brenton S, Janus ED, Knight K. Alpha-1-antitrypsin PLowell: a normally functioning variant present in low concentration. Aust NZ J Med 1995:25:695-7.

12. Huet-Duvillier G, Balduyck M, Watrigant Y, et al. Relationship between a mild alpha 1 proteinase inhibitor deficiency and respiratory symptoms in a family. Ann Clin Biochem 1995;32:545-9.

13. Cook L, Knight K, Burdon JG, Brenton S, Hunt JM. Function of a rare variant of alpha-1 antitrypsin, phenotype $\mathrm{P}(\mathrm{i})$ EFranklinS, a poor inhibitor of human neutrophil elastase. Res Exp Med 1997:196:355-62. 


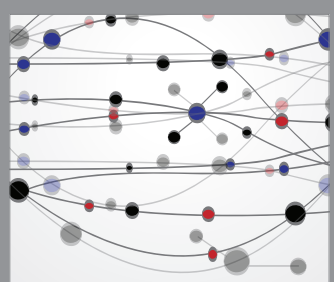

The Scientific World Journal
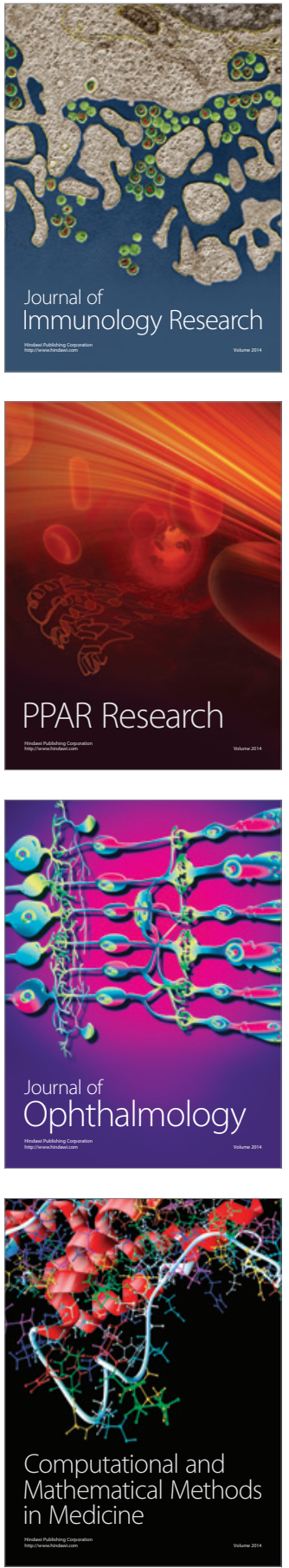

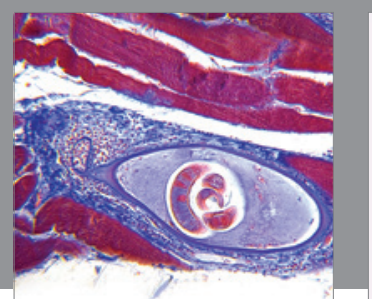

Gastroenterology Research and Practice

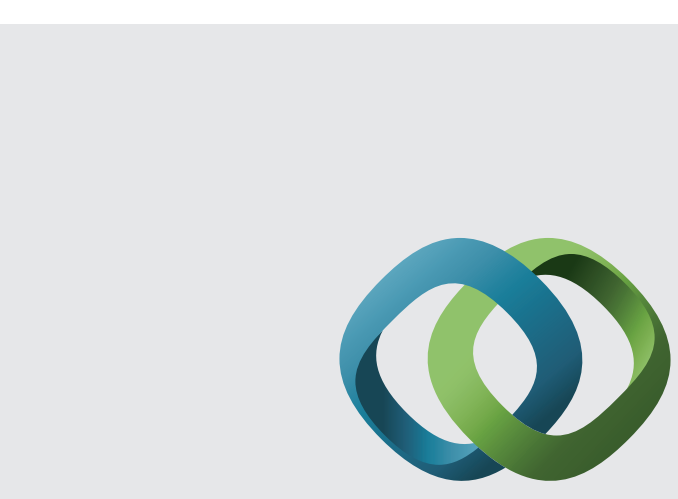

\section{Hindawi}

Submit your manuscripts at

http://www.hindawi.com
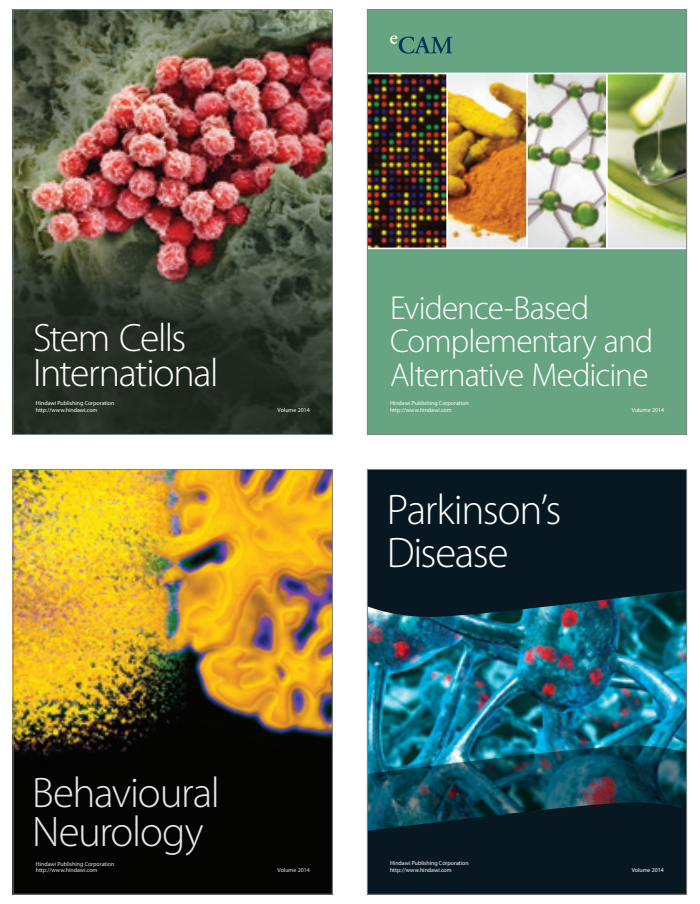
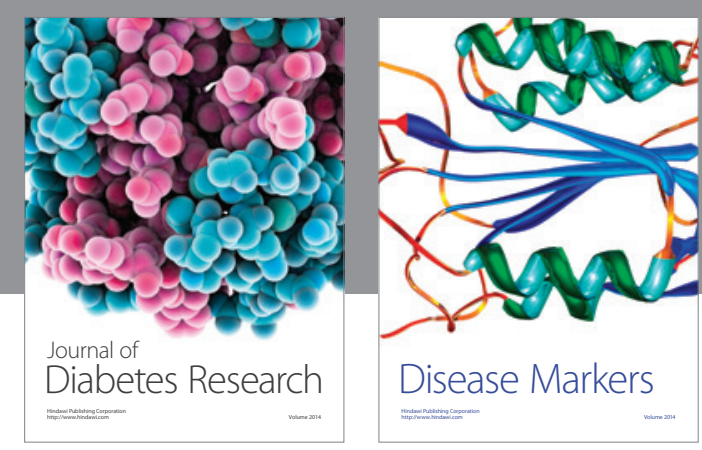

Disease Markers
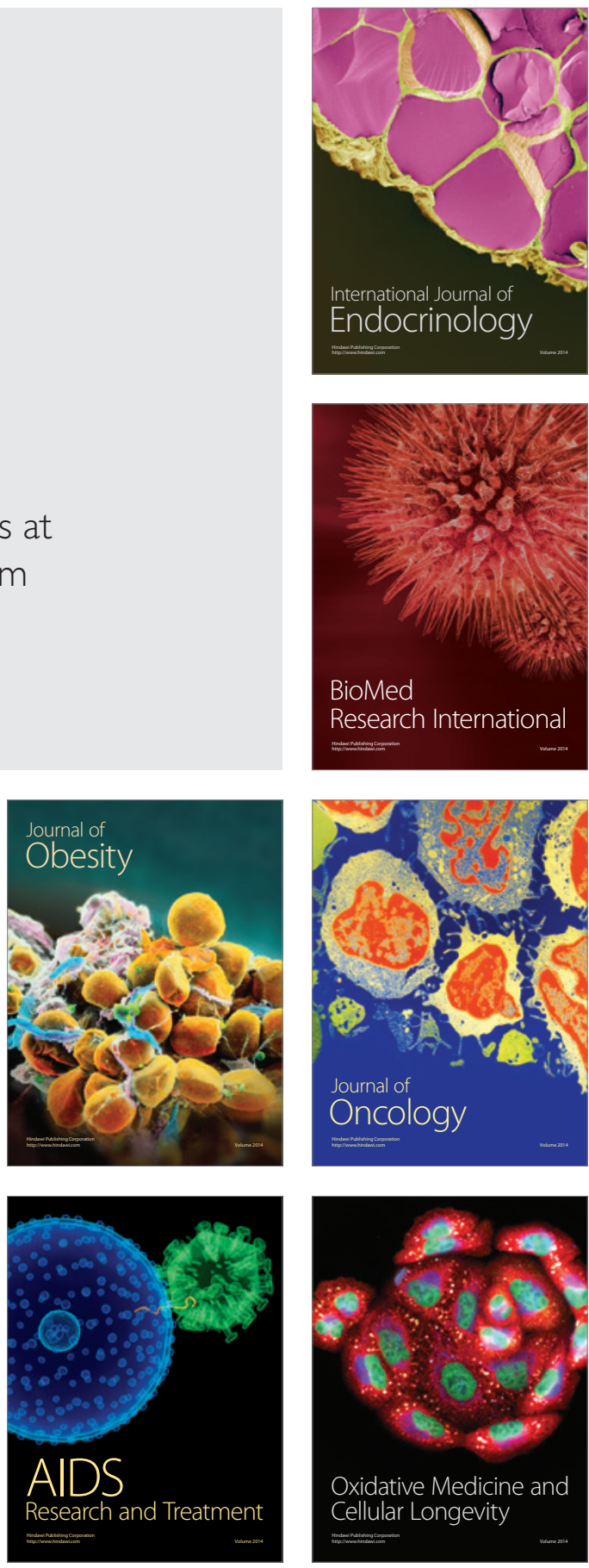